\title{
Caracterização de nanopartículas poliméricas preenchidas com óleo essencial de Piper nigrum por microscopia de força atômica
}

\author{
Characterization of polymeric nanoparticles \\ filled with Piper nigrum essential oil by \\ atomic force microscopy
}

\author{
Ítalo Carvalho Costa ${ }^{1}$, Sidney Gomes Azevedo ${ }^{2}$, Edgar Aparecido Sanches ${ }^{2}$,
} Henrique Duarte da Fonseca Filho ${ }^{1}$

\begin{abstract}
${ }^{1}$ Universidade Federal do Amazonas - UFAM, Laboratório de Síntese de Nanomateriais e Nanoscopia LSNN, Av. General Rodrigo Octávio, 6200, Coroado, CEP: 69067-005, Manaus, AM, Brasil

${ }^{2}$ Universidade Federal do Amazonas - UFAM, Laboratório de Polímeros Nanoestruturados- NANOPOL-Av. General Rodrigo Octávio, 6200, Coroado. CEP: 69067-005, Manaus, AM, Brasil

e-mail: italo.carvalhocosta@gmail.com, hdffilho@ufam.edu.br, sidneyazevedo1@gmail.com, sanches.ufam@gmail.com
\end{abstract}

\begin{abstract}
RESUMO
Óleos essenciais derivados de plantas têm chamado a atenção devido a uma série de propriedades físicoquímicas e atividades biológicas. Entretanto, eles possuem diversas limitações, pois são voláteis e instáveis na presença de umidade, altas temperaturas, ar e luz e, assim, técnicas de encapsulamento surgem como alternativa para proteção das propriedades funcionais desses óleos fornecendo, ainda, uma liberação lenta e controlada. Devido a lenta degradação biológica dos materiais que compõem a parede da cápsula e a duração prolongada da ação de liberação do óleo, micro e nanocápsulas são de grande interesse científico e tecnológico. Neste trabalho, nanopartículas à base de bicamada foram desenvolvidas usando gelatina e poli-ecaprolactona visando proteger o óleo essencial de Piper nigrum por meio de encapsulamento e analisadas por microscopia de força atômica. Nanopartículas com e sem óleo essencial de pimenta preta foram preparadas e gotejadas em lâminas de vidro para formar filmes que foram investigados por imagens com resoluções de 256 x 256 pixels com áreas de $5 \times 5 \mathrm{~m}^{2}$. O óleo foi obtido por meio de sementes de pimenta que foram tritu-

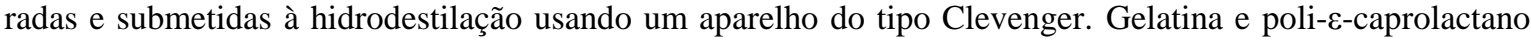
foram usados como material de parede, cuja eficiência de encapsulamento foi de 98,50\%. Foram obtidas

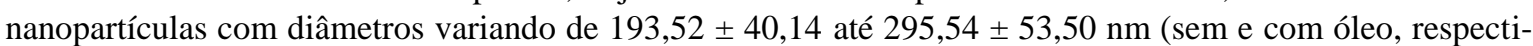
vamente). Além disto, foram comparadas também as alturas, densidades e o desvio médio quadrático das rugosidades das amostras indicando uma tendência de aumento nestes parâmetros em função da incorporação de óleo essencial na síntese, exceto na densidade devido ao crescimento das outras dimensões.
\end{abstract}

Palavras-chave: Pimenta preta. Óleo essencial. Nanoencapsulamento. Microscopia de força atômica. Morfologia.

\footnotetext{
ABSTRACT

Essential oils derived from plants have attracted attention due to a several physico-chemical properties and biological activities. However, they have several limitations, as they are volatile and unstable in the presence of humidity, high temperatures, air and light and, thus, encapsulation techniques appear as an alternative to protect the functional properties of these oils while still providing a slow and controlled release. Due to the slow biological degradation of the materials that make up the capsule wall and the prolonged duration of the oil release action, micro and nanocapsules are of great scientific and technological interest. In this work, bilayer-based nanoparticles were developed using gelatin and poly- $\varepsilon$-caprolactone to protect the essential oil of Piper nigrum through encapsulation and analyzed by atomic force microscopy. Nanoparticles with and without black pepper essential oil were prepared and dripped onto glass slides to form films that were investigat-
} 
ed by images with resolutions of 256 x 256 pixels with areas of $5 \times 5 \mu \mathrm{m}^{2}$. The oil was obtained through pepper seeds that were crushed and subjected to hydro distillation using a Clevenger type apparatus. Gelatin and poli- $\varepsilon$-caprolactane were used as wall material to increase the size of the nanoparticles and to obtain greater protection from the OE, resulting in an encapsulation efficiency of $98.50 \%$. Nanoparticles with diameters ranging from $193.52 \pm 40.14$ to $295.54 \pm 53.50 \mathrm{~nm}$ (without and with oil, respectively) were obtained. In addition, heights, densities and, average and root mean square roughness of the sample images were also compared, indicating a tendency of increase in these parameters due to the incorporation of essential oil in the synthesis, except in density due to the growth of other dimensions.

Keywords: Black pepper. Essential oil. Nanoencapsulation. Atomic force microscopy. Morphology.

\section{INTRODUÇÃO}

O gênero Piper, pertencente à família Piperaceae, consiste em cerca de 2.000 espécies [1] e, dentre elas, destaca-se a pimenta preta (Piper nigrum), uma das especiarias mais usadas do mundo [2]. Assim como as sementes da $P$. nigrum, conhecida popularmente como pimenta do reino, o óleo essencial (OE) desta espécie também apresenta uma infinidade de atividades biológicas e tem sido tradicionalmente usado em diferentes aplicações, incluindo tratamento de várias doenças devido aos seus componentes químicos [3], sendo rico em monoterpenos e sesquiterpenos que variam de $1,2 \%$ a $3,5 \%$ de sua totalidade [4-6].

Conforme ABDULAZEEZ et al. [5], o OE de Piper nigrum desempenha um papel significativo no processamento de alimentos, saúde e nutrição ao prevenir ou retardar a deterioração de alimentos devido às propriedades antioxidantes e antimicrobianas apresentadas por alguns de seus constituintes que se sobressaem. Esse $\mathrm{OE}$ é uma mistura de um grande número de compostos químicos voláteis e devido à grande diversidade química de componentes, este óleo apresenta uma gama de atividades biológicas, bem como: atividade antifúngica, anti-inflamatória [7], antimicrobiana [8], antioxidante [9] e até mesmo atividade larvicida [10], tornando-se uma boa alternativa de compostos naturais a serem utilizados pela indústria farmacêutica. No entanto, esses óleos são voláteis e quimicamente instáveis na presença de ar, luz, umidade e altas temperaturas,e sua rápida degradação pode ser um empecilho para determinadas aplicações. Com isso, a nanoencapsulação do OE é um procedimento promissor, fornecendo uma boa proteção do agente ativo e uma liberação lenta e controlada.

Sucintamente, as vantagens da nanoencapsulação de OEs são: facilidade de manuseio, estabilidade, proteção contra oxidação, melhor distribuição, solubilidade, liberação controlada e aumento da área de superfície por unidade de volume [8]. Os veículos comumente preparados para encapsulamento de OE são as nanopartículas lipídicas sólidas (NLS), lipossomos e as nanopartículas poliméricas (NPP) [11]. De acordo com SÃO PEDRO et al. [12], o encapsulamento de OE em NPPs apresenta vantagens, como: liberação controlada, maior solubilidade em água, citotoxicidade reduzida e atividade antimicrobiana aprimorada.

As NPPs são sistemas coloidais compostos por polímeros naturais, sintéticos ou semissintéticos. Considerando os mecanismos de encapsulamento, o OE pode ser aprisionado, disperso, dissolvido dentro ou adsorvido nas nanopartículas e, em geral, seu diâmetro médio está na faixa de 50 a $1000 \mathrm{~nm}$ [13]. BANIK et al. [14] relatam que as NPPs são uma das estratégias orgânicas mais estudadas para nanomedicina. O uso de materiais biodegradáveis baseados em nanotecnologia para combater pragas representa uma área interessante de pesquisa e, para PASCOLI et al. [15] esses nanoprodutos são adequados para armazenar quantidades muito pequenas de agrotóxicos, devido à sua biocompatibilidade, biodegradabilidade e baixa toxicidade, podendo transportar muitas classes de agroquímicos, incluindo herbicidas, inseticidas, fungicidas, acaricidas, e fertilizantes [15].

Diferentes tipos de materiais de parede e tensoativos são usados com vários métodos para criar o encapsulamento de OE com diferentes estruturas, capacidades e perfis de liberação [16]. Para uma síntese de nanopartículas biodegradáveis, é necessário procurar um método verde de síntese e uso de estabilizadores que sejam biogênicos e não tóxicos para o desenvolvimento do sistema de liberação $[17,18]$. Materiais de parede, como os polímeros quitosana, ciclodextrina, albumina e gelatina, têm sido utilizados nos diferentes sistemas de entrega para proteger os OEs de fatores intrínsecos e extrínsecos como pH, atividade da água, degradação enzimática, temperatura, umidade relativa e ambiente de armazenamento [19].

Dentre os diversos polímeros utilizados como material de parede de NPPs tem-se um grande interesse na gelatina e na poli- $\varepsilon$-caprolactano (PCL), ambas aprovadas pela Food and Drug Administration (FDA). Isto se deve ao fato que a gelatina é biodegradável, não-tóxica, fácil de reticular e tem um imenso potencial a ser usado para a preparação de sistemas coloidais de liberação de fármacos [20, 21]. Já o PCL é um excelente polímero para encapsulação de OE por ser um poliéster alifático semicristalino, que é considerado um material biocompatível e bio-reabsorvível com alta permeabilidade às drogas [22]. A gelatina, que pode ser de três 
tipos (tipo A, obtido de fontes de suínos, tipo B obtido de origem bovina e um terceiro tipo proveniente de fontes marinhas [22]), é obtida pela hidrólise do colágeno, que é o principal componente da pele, ossos e tecido conjuntivo [20].

As NPPs também podem ser formadas promovendo a autoassociação ou agregação de biopolímeros, podendo levar como componentes funcionais, vitaminas, agentes antimicrobianos e muitos outros componentes diferentes [23]. A associação de polímeros como materiais de parede está diretamente ligada ao tamanho de NPPs que se formam por meio desta junção, acarretando modificações em todo sistema.

Um agente que também tem um papel fundamental em muitas formulações de nanopartículas biopoliméricas é o emulsificante, agente de interface, tensoativo, conhecido também como surfactante. Os agentes emulsificantes têm como função reduzir a tensão interfacial, facilitando a fragmentação em partículas menores e agindo contra a coalescência das gotículas [24]. Os surfactantes das séries TWEEN e SPAN são comuns para estabilização de nanoemulsões [25]. O monoestearato de sorbitano (SPAN 60) é um éster obtido pela reação de sorbitano (derivado do sorbitol) com o ácido esteárico, e é um tensioativo não iônico que apresenta um valor de Balanço Hidrofílico-Lipofílico (HBL) de 4,7. Já o Monolaurato de polioxietilenosorbitano (TWEEN 80), é um agente resultante da reação de um éster de sorbitano com o óxido de eteno, é um tensioativo não iônico, solúvel em água e com um valor de BHL de 15,0 [26].

Com o avanço da nanociência e da nanotecnologia, surgiu uma grande variedade de técnicas analíticas para a caracterização de sistemas em escala micro e nanométrica [27] e, dentre elas a microscopia de força atômica (AFM) ganha destaque na caracterização de NPPs pois ela permite produzir mapas de imagens topográficas tridimensionais com resolução na escala nano, podendo ainda ser usada na investigação de características físicas, tais como elétrica e magnética, e propriedades mecânicas de sistemas particulados. Froiio et al. produziram e investigaram, em termos de tamanho e distribuição de tamanho, NPPs com OEs de laranja e bergamota, usando imagens de AFM [28]. Estudos de NPPs que também tinham como materiais de parede PCL/gelatina, mas envolvendo OEs de outras plantas, tais como o alho (Allium sativum) e as pimentas das espécies Piper aduncum e Piper hispidinervum, também foram analisadas por AFM [21, 29].

A compreensão das características físicas das NPPs é crucial para o entendimento do carreamento, das condições ambientais suportáveis, para prever o comportamento e a integridade durante o manuseio, armazenamento e transporte. Isto porque as NPPs usadas como transportadores de bioativo devem ser projetadas para se romperem comedidamente sob o estímulo de uma mudança nas condições ambientais, sendo capaz de entregar uma medida ponderada do bioativo ao alvo. A investigação das características físicas desses nanotransportadores através de AFM se dá por meio de uma abordagem morfológica completa. Neste artigo, a eficiência de encapsulamento do ativo e os aspectos morfológicos tais como: tamanho, forma, distribuição, densidade e altura das NPPs são apresentados.

\section{MATERIAIS E MÉTODOS}

\subsection{Extração do óleo essencial de Piper nigrum}

As sementes de Piper nigrum (SisGen $\mathrm{n}^{\circ}$ A26CD5E), mostradas na Figura 1, foram obtidas em mercado local da cidade de Manaus-AM, sendo cuidadosamente selecionadas e lavadas com água destilada para remover as impurezas e depois secas em um forno a uma temperatura não superior a $40{ }^{\circ} \mathrm{C}$. Após a secagem, as sementes foram armazenadas em um frasco selado, longe da luz e da umidade até o momento da extração do OE.

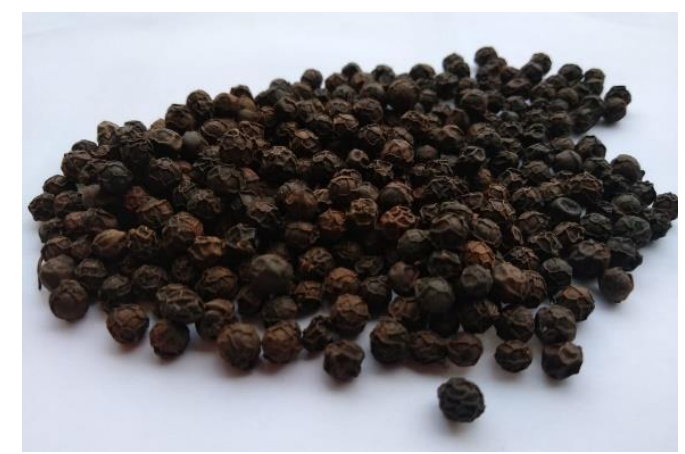

Figura 1: Foto das sementes de Piper nigrum. 
Uma porção de $300 \mathrm{~g}$ de semente, após trituração, foi submetida à hidrodestilação usando um aparelho do tipo Clevenger modificado por 4 horas a $100^{\circ} \mathrm{C}$ [9]. Após a extração, o volume do OE foi quantificado com o auxílio de uma micropipeta, armazenado em um frasco âmbar e mantido a $-18^{\circ} \mathrm{C}$, conservando desta forma os seus constituintes e suas propriedades biológicas.

\subsection{Síntese de nanopartículas poliméricas carreadas}

As NPPs podem ser obtidas por diferentes métodos, inclusive uma combinação dos mesmos, levando o desenvolvimento de veículos eficientes para formulações contendo OE. A gelatina e a PCL foram utilizados como material de parede para o aumento de tamanho das nanopartículas e para obter maior proteção do OE, sendo essencial o uso da transglutaminase para ligar os dois polímeros no encapsulamento. TACC, SPAN 60 e TWEEN 80 foram utilizados como surfactantes e o conservante de fenoxietanol/2-metil-2H-isotiazolin-3ona (NE) para aumentar a durabilidade dessas nanopartículas. A Tabela 1 apresenta os componentes utilizados no desenvolvimento desta formulação, bem como suas concentrações.

Tabela 1: Concentrações utilizadas para $150 \mathrm{~mL}$ de síntese.

\begin{tabular}{l|c|c}
\hline \multirow{4}{*}{ FASES } & COMPONENTES & CONCENTRAÇÃo \\
\hline \multirow{4}{*}{ Argânica } & Gelatina tipo B & $1,0 \mathrm{~g}$ \\
\cline { 2 - 3 } & TWEEN 80 & $0,3 \mathrm{~g}$ \\
\cline { 2 - 3 } & Água destilada & $150 \mathrm{~mL}$ \\
\cline { 2 - 3 } & PCL & $0,05 \mathrm{~g}$ \\
\cline { 2 - 3 } & Óleo essencial & $1000 \mu \mathrm{g} . \mathrm{mL}^{-1}$ \\
\cline { 2 - 3 } & SPAN 60 & $0,02 \mathrm{~g}$ \\
\cline { 2 - 3 } & TACC & $0,1 \mathrm{~g}$ \\
\hline Agente de ligação & Diclorometano & $10 \mathrm{~mL}$ \\
\hline Conservante & Transglutaminase & $0,0935 \mathrm{~g}$ \\
\hline
\end{tabular}

O preparo da síntese inicia aquecendo a gelatina tipo B a $50{ }^{\circ} \mathrm{C}$ em água destilada sob agitação constante (solução I) e, em outro béquer, o surfactante TWEEN 80 foi solubilizado em água destilada à $25^{\circ} \mathrm{C}$ (solução II). Após a solução I resfriar, atingindo $43^{\circ} \mathrm{C}$, verteu-se a mesma na solução II. Este procedimento inicial é conhecido como Fase Aquosa. Em outros dois béqueres, a PCL (solução III), o emulsificante SPAN 60 e o TACC - Triglicérides do Ácido Cáprico e Caprílico (solução IV) foram solubilizados em diclorometano. Esta segunda etapa de solubilização é a chamada Fase Orgânica. Nesta, para as sínteses de nanopartículas preenchidas, foi adicionado o OE. Previamente solubilizadas, verteu-se a solução III na solução IV.

Em seguida, a fase orgânica foi adicionada à fase aquosa usando um ultra-dispersor (10.000 rpm) por 30 segundos. E, sob agitação constante $\left(25^{\circ} \mathrm{C}\right)$, a transglutaminase foi acrescentada à solução final, permanecendo sob agitação até evaporação total do solvente. $\mathrm{O}$ pH final da síntese foi ajustado para 8,0, sendo a solução filtrada e armazenada e foi adicionado o conservante NE nas amostras que seriam avaliadas ao longo do tempo. A Figura 2 traz uma representação esquemática da metodologia da síntese. 


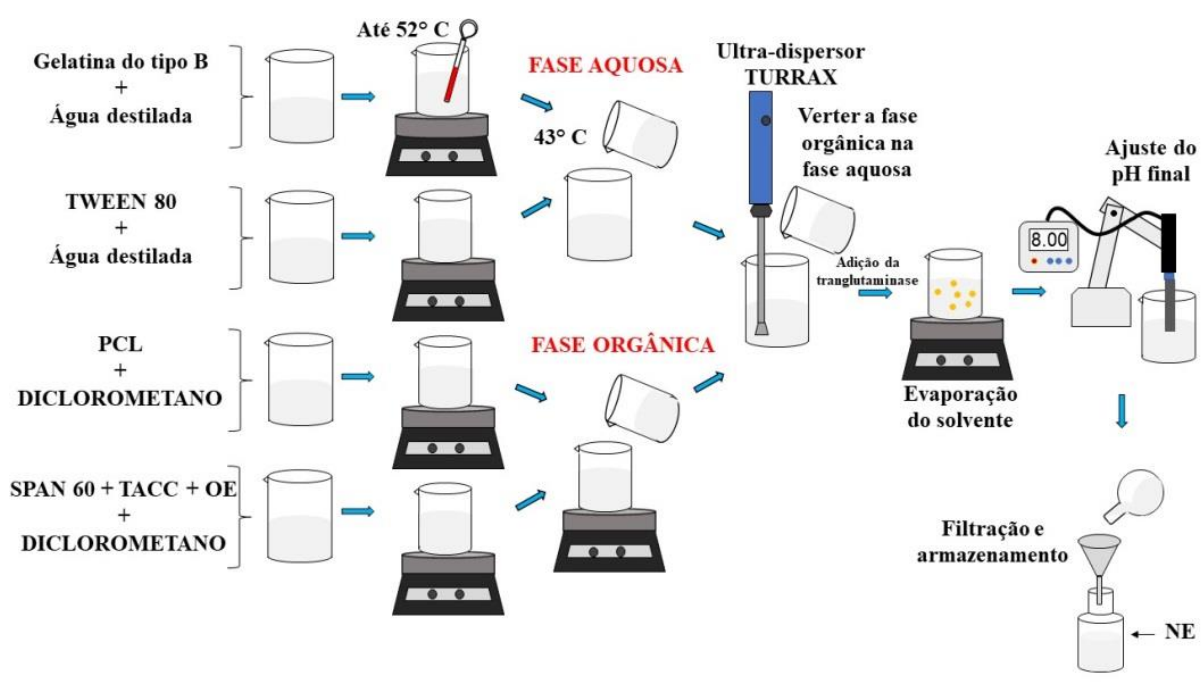

Figura 2: Representação esquemática da formulação de nanopartículas.

\subsection{Eficiência de encapsulamento por UV-vis}

Para estimar a eficiência de encapsulamento (EE) dos OEs (\%) nas NPPs biodegradáveis de gelatina e PCL, foi utilizado um espectrofotômetro UV-Vis (Global Trade Technology), com base no trabalho de GHASEMISHAHRESTANI et al. (2015) [20] com algumas modificações. Inicialmente, foi realizada uma varredura com o OE da Piper nigrum, obtendo o pico máximo de absorção em $\lambda=229 \mathrm{~nm}$. A curva de calibração foi previamente desenvolvida com diferentes concentrações do ativo em metanol (1,9 a $1000 \mathrm{mg}$. $\left.\mathrm{mL}^{-1}\right)$, para qual se mostrou ser altamente solúvel. As soluções foram analisadas em triplicata e as médias das áreas dos picos referentes a cada concentração foram ajustadas em um gráfico de absorbância versus concentração. A equação da reta e o coeficiente de correlação linear foram calculados pela análise da regressão linear para a obtenção da curva de calibração, que é apresentada na Figura 3. O OE encapsulado nos transportadores foi separado dentro da dispersão coloidal. A dispersão fornecida foi centrifugada a $10.000 \mathrm{rpm}$ (DAIKI, modelo DTR20.000) por 10 min e a absorbância do sobrenadante foi usada para determinar a quantidade de OE livre.

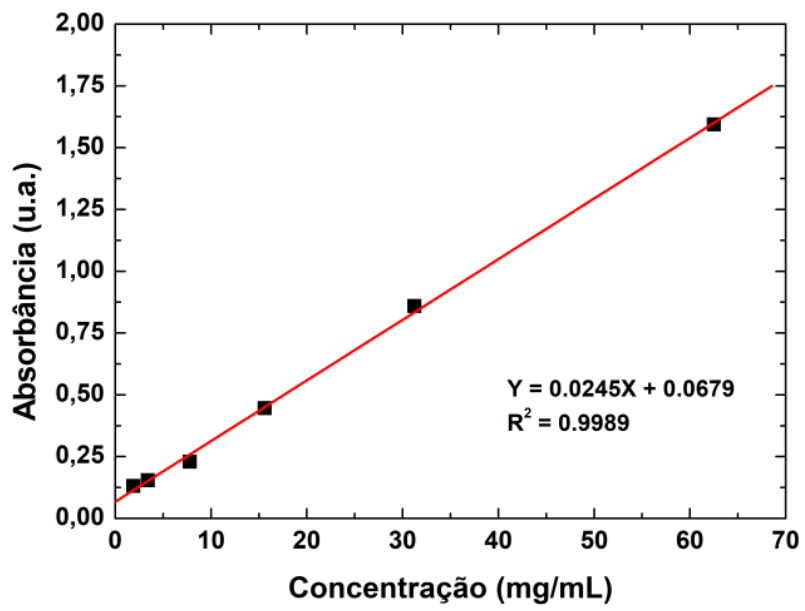

Figura 3: Curva de calibração do óleo essencial de Piper nigrum

\subsection{Microscopia de força atômica - AFM}

As imagens de topografia das NPPs foram obtidas com um AFM modelo Innova (Bruker), operando em modo tapping, equipado com uma ponta de silicone e cantilever revestido de $\mathrm{Al}$ e com uma constante de mola de 42 N/m (Tap190AL-G da BudgetSensorsTM, Sofia, Bulgária). As varreduras foram realizadas em áreas de $5 \times 5$ e 10x10 $\mu \mathrm{m}^{2}$ com 512x512 pixels a uma taxa de varredura de $1 \mathrm{~Hz}$. As medidas foram realizadas à temperatura ambiente $\left(23 \pm 1{ }^{\circ} \mathrm{C}\right)$ e $60 \pm 1 \%$ de umidade relativa. O controle de realimentação foi ajustado à superfície das amostras para obter as melhores imagens possíveis. As imagens de topografia foram ajustadas 
no plano, para ajuste de iluminação, e a altura média das linhas da imagem foi ajustada com um filtro "flatten" de ordem zero, usando o software Gwyddion, versão 2.51 [30]. Além disso, o software imageJ foi usado com base no estudo das aplicações de tratamento de imagens segundo SCHNEIDER et al. (2012) [31], para a obtenção de alguns parâmetros relacionados a morfologia das NPPs. Para obter os resultados morfológicos, os sistemas coloidais contendo as nanopartículas não carregadas ou carregadas $(1 \mu \mathrm{L})$ foram gotejados em uma lâmina de vidro e secos usando nitrogênio líquido. Em seguida, as lâminas de vidro contendo os filmes formados foram fixadas no porta-amostra do AFM usando uma fita adesiva dupla face.

\section{RESULTADOS E DISCUSSÃO}

\subsection{Eficiência de encapsulamento}

Logo após a síntese das nanopartículas, calculou-se a eficiência de encapsulamento (EE) para uma concentração absoluta de $1000 \mu \mathrm{g} . \mathrm{mL}^{7}$, com base no trabalho de Silva et al. [21], considerando a razão da quantidade de OE da Piper nigrum e o total de OE medido em suspensão do sistema. A EE foi então obtida usando a fórmula: \% de eficiência de encapsulamento $(\mathrm{EE})$ = (quantidade de óleo essencial encapsulado / quantidade total de óleo essencial usado na formulação) *100, sendo um dos parâmetros de qualidade utilizados para determinar a quantidade de óleo encapsulado com sucesso pela secagem por pulverização. Foi obtido um valor de eficiência de encapsulamento de $98,50 \%$, que é superior a diversos trabalhos que também utilizaram polímero como material de parede no encapsulamento de OEs, inclusive de outras espécies de Piper. YANG et al. [32], encontraram um valor de $\mathrm{EE}=80 \%$ em nanopartículas preenchidas de alho, que tinham como material de parede o polietileno glicol (PEG). BADRI et al. [33] mostraram um valor de EE = 84\% no encapsulamento de OE de Nigella Sativa L. em nanopartículas biodegradáveis de PCL. GHASEMISHAHRESTANI et al. [20] encontraram um valor de $\mathrm{EE}=97 \%$ em nanopartículas preenchidas de quercetina, tendo como material de parede a gelatina. SILVA et al. [21] apresentaram um valor de EE =79,2\% no encapsulamento de OE de Piper aduncum e OE de Piper hispidinervum em nanopartículas biodegradáveis de gelatina. Após três meses da data de sintetização, as NPPs preenchidas de OE apresentaram um valor de encapsulamento de 93,00 \%, um valor maior em relação aos trabalhos citados anteriormente (medidos logo após o término da síntese), ilustrando também que o conservante usado neste trabalho está bastante adequado.

\subsection{Análise morfológica via AFM}

As figuras 4(a) e 4(b) apresentam imagens da superfície dos filmes poliméricos com as nanopartículas com e sem óleo essencial, respectivamente e diferenças podem ser observadas, tanto na altura quanto no diâmetro das nanocápsulas, bem como a distribuição delas sobre a superfície devido à incorporação do óleo à síntese.

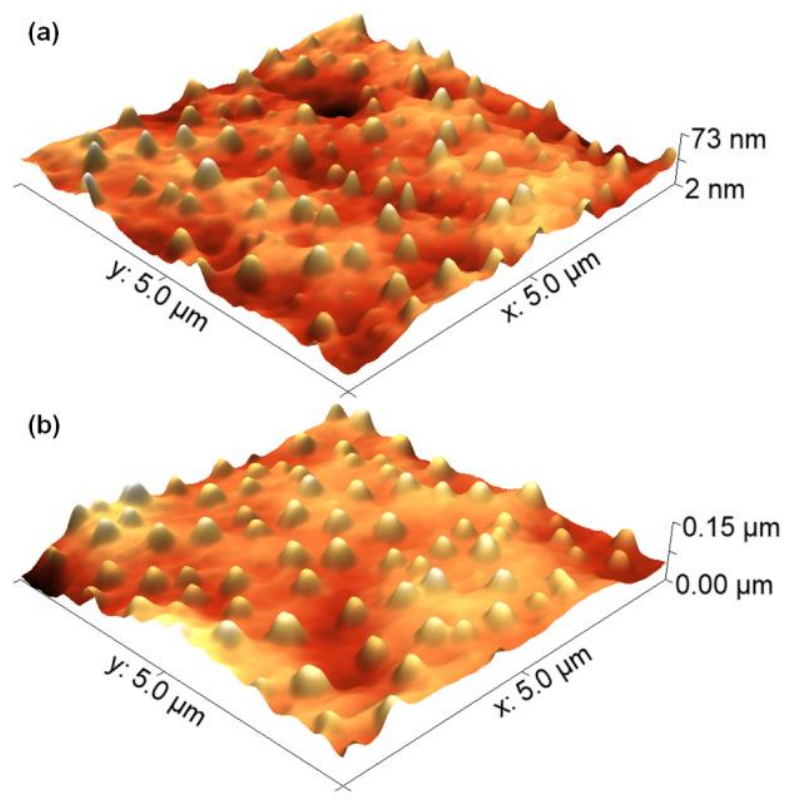

Figura 4: Imagens de altura obtidas com o AFM das (a) nanopartículas sem OE e (b) com OE de P. nigrum. 
Com as imagens topográficas da figura 4, nota-se que as NPPs apresentam geometria próxima a esférica e que a distribuição desse sistema é uniforme. Apesar do tamanho das NPPs possuírem valores aproximados, elas podem estar distribuídas de forma mais dispersa ou mais aglomerada. As regiões mais claras das imagens referem-se a domínios mais altos e ordenados da membrana gelatina/PCL. Através do software ImageJ foi possível processar as imagens e calcular os valores médios de diâmetro e densidade [31], enquanto que os valores de altura foram extraídos do software Gwyddion, cujos resultados estão na Tabela 2. O diâmetro médio das NPPs preenchidas com OE foi de 295,54 \pm 53,50 nm e das NPPs sem o OE 193,52 \pm 40,14 nm, e essa variação nos valores ocorre devido ao encapsulamento do ativo. No entanto, foi possível detectar que essa diferença de tamanho é razoável. Sabe-se que o tamanho dessas NPPs depende não somente da concentração do ativo, mas também do método de preparação e dos materiais de parede [23]. NPPs foram obtidas numa faixa de tamanho de 140 a $190 \mathrm{~nm}$ usando o método de nanoemulsão [34] e uma faixa de 30 - $40 \mathrm{~nm}$ usando a nanoprecipitação [35], ambas tendo a gelatina como material de parede. Além disso, também foi observado um aumento considerável nas alturas médias das nanocápsulas carregadas em comparação com as vazias, conforme apresenta a tabela 2. Desta forma, devido tanto ao aumento no diâmetro quanto nas alturas médias, percebe-se que há uma diminuição na densidade de NPPs (distribuição de nanocápsulas por $\mu m^{2}$ ) que provavelmente ocorre em função de um rearranjo delas ao incorporarem o OE. Para toda esta análise morfológica, cinco imagens tomadas em diferentes pontos das amostras foram usadas para fins de cálculo dos parâmetros comentados anteriomente, além dos valores de rugosidade RA e RMS.

Tabela 2: Parâmetros relacionados à morfologia das nanopartículas.

\begin{tabular}{c|c|c|c}
\hline AMOSTRA & DIÂMETRO $(\mathbf{n m})$ & DENSIDADE $\left(\right.$ nanocápsulas/ $\left.\boldsymbol{\mu m ^ { 2 }}\right)$ & ALTURA $(\mathbf{n m})$ \\
\hline Sem óleo & $193,52 \pm 40,14$ & $2,92 \pm 0,08$ & $41,30 \pm 3,37$ \\
\hline Com óleo & $295,54 \pm 53,50$ & $2,51 \pm 0,05$ & $66,90 \pm 3,41$ \\
\hline
\end{tabular}

A rugosidade média aritmética da superfície (Ra) é um dos parâmetros mais usados universalmente e é definido como o desvio médio absoluto de irregularidades na rugosidade da linha média ao longo de um comprimento da superfície. Ra é obtido através da equação (1), em que [36]:

$$
R_{a}=\frac{1}{N_{x} N_{y}} \sum_{i=1}^{N_{x}} \sum_{j=1}^{N_{y}}\left|z(i, j)-z_{\text {médio }}\right|
$$

onde

$$
z_{\text {médio }}=\frac{1}{N_{x} N_{y}} \sum_{i=1}^{N_{x}} \sum_{j=1}^{N_{y}} z_{i j}
$$

e $\mathrm{N}_{\mathrm{x}}$ e $\mathrm{N}_{\mathrm{y}}$ representam o número de pontos nos eixos x e $\mathrm{y}$, respectivamente. A rugosidade quadrada média $\mathrm{R}_{\mathrm{q}}$ é o desvio padrão da distribuição da altura da superfície e é usado para descrever a rugosidade da superfície usando métodos estatísticos, sendo encontrada pela equação [36]:

$$
R_{q}=\sqrt{\frac{1}{N_{x} N_{y}} \sum_{i=1}^{N_{x}} \sum_{j=1}^{N_{y}}\left(z(i, j)-z_{m e ́ d i o}\right)^{3}}
$$

A Tabela 3 apresenta uma análise da influência do encapsulamento de OE na rugosidade das nanopartículas. Estes valores de rugosidades foram extraídos do filme de nanopartículas preenchidas e vazias, após a secagem no substrato.

Tabela 3: Rugosidade das nanopartículas.

\begin{tabular}{c|c|c}
\hline \multirow{2}{*}{ AMOSTRA } & \multicolumn{2}{|c}{ RUGOSIDADE $(\mathbf{n m})$} \\
\cline { 2 - 3 } & RMS & RA \\
\hline Sem óleo & $11,49 \pm 1,66$ & $8,720 \pm 1,14$ \\
\hline Com óleo & $19,65 \pm 1,80$ & $14,74 \pm 0,99$ \\
\hline
\end{tabular}


De acordo com os valores encontrados, verifica-se que ambas as rugosidades (RMS) e (RA) seguem uma tendência de aumento em razão da presença do OE no interior das nanocápsulas. Estes resultados confirmam o que já foi relatado anteriormente em relação à morfologia das superfícies dos filmes e das respectivas cápsulas. O filme seco de NPPs preenchidas, além de contar com cavidades e elevações de materiais nos substratos por causa dos biopolímeros que se rompem, conta também com a presença dos constituintes do ativo liberado parcialmente.

\section{CONCLUSÕES}

O presente trabalho desenvolveu com sucesso nanopartículas à base de gelatina que serviram como transportadoras para o OE da espécie $P$. nigrum. As nanopartículas carregadas desenvolvidas apresentaram uma alta eficiência de encapsulamento para a concentração absoluta de $1.000 \mu \mathrm{g} \mathrm{mL}^{-1}$ de OE, que foi de $98,50 \%$, permanecendo após três meses acima de $90 \%$. Sínteses de NPPs com e sem OE foram preparadas e gotejadas sobre lâminas de vidro, formando filmes cujas superfícies foram analisadas por microscopia de força atômica (AFM). Os resultados de AFM mostraram que o incorporamento de óleo essencial na síntese aumenta o tamanho das cápsulas e assim, os diâmetros das NPPs variaram de 193,52 \pm 40,14 a 295,54 \pm 53,50 nm, exibindo perfis de distribuição semelhantes, independentemente da inclusão de OE. A variação nas alturas e a quantidade de nanocápsulas por $\mu \mathrm{m}^{2}$ também foi estudada, onde as cápsulas com óleo são mais altas e um pouco menos densas. Verificou-se ainda como esses parâmetros influenciaram os valores de rugosidade RMS e RA, que apresentaram aumento devido a presença de óleo. Portanto, este artigo fornece informações morfológicas importantes para sistemas de nanopartículas de gelatina e PCL preenchidas com OE de Piper nigrum, não existente até então em literatura, podendo servir como referência também para outros sistemas de nanotransportadores.

\section{AGRADECIMENTOS}

Os autores agradecem a Fundação de Amparo à Pesquisa do Amazonas (FAPEAM) pelo apoio financeiro concedido.

\section{BIBLIOGRAFIA}

[1] GULZAR, T., et al., "New constituents from the dried fruit of Piper nigrum Linn., and their larvicidal potential against the Dengue vector mosquito Aedes aegypt". Phytochemistry Letters, v. 6, n.2, pp. 219-223, Feb. 2013.

[2] ZHU, F., MOJEL, R., LI, G.,"Structure of black pepper (Piper nigrum) starch”. Food Hydrocolloids, v. 71, n. 1, pp. 102-107, Oct. 2017.

[3] CARNEVAlli, D.B., ARAÚJO, A.,“Atividade Biológica da Pimenta Preta (Piper nigrun L.): Revisão de Literatura. UNICIÊNCIAS, v. 17, n. 1, pp. 41-46, Dec. 2013.

[4] TRAN, T.H., et al., "The Study on Extraction Process and Analysis of Components in Essential Oils of Black Pepper (Piper nigrum L.) Seeds Harvested in Gia Lai Province, Vietnam”. Processes, v. 7, n. 2, pp. 116, Jan. 2019.

[5] ABDULAZEEZ, M.A. et al., "Black Pepper (Piper nigrum L.)", In: Victor R. Preedy, Essential Oils in Food Preservation, Flavor and Safety, v. 1, Elsevier, pp. 277 - 285, 2016.

[6] ZHAI, H., et al., "Potential of essential oils for poultry and pigs". Animal Nutrition, v. 4, n. 2, pp. 179186, Jun. 2018.

[7] AHMAD, N., et al., "Biological role of Piper nigrum L. (Black pepper): A review". Asian Pacific Journal of Tropical Biomedicine, v. 2, n. 3, pp. 1945-1953, Dec. 2012.

[8] PRAKASH, B., et al., "Nanoencapsulation: An efficient technology to boost the antimicrobial potential of plant essential oils in food system". Food Control, v. 89, n. 1, pp. 1-11, Jun. 2018.

[9] BAGHERI, H., et al., "Antioxidant activity of Piper nigrum L. essential oil extracted by supercritical 
CO2 extraction and hydro-distillation". Talanta, v. 121, n. 1, pp. 220-228, Apr. 2014.

[10] SAMUEL, M., et al., "The larvicidal effects of black pepper (Piper nigrum L.) and piperine against insecticide resistant and susceptible strains of Anopheles malaria vector mosquitoes". Parasites \& Vectors, v. 9, n. 238, pp. 1-9, Apr. 2016.

[11] ASBAHANI, A.E., et al., "Essential oils: From extraction to encapsulation". International Journal of Phar-maceutics, v. 483, n. 1, pp. 220-243, Feb. 2015.

[12] SÃO PEDRO, A., et al., The use of nanotechnology as an approach for essential oil-based formulations with antimicrobial activity, 1 ed., chapter 2, Bahia, Formatex, 2013.

[13] GUTERRES, S.S., ALVES, M.P., POHLMANN, A.R.,"Polymeric Nanoparticles, Nanospheres and Nanocapsules, for Cutaneous Applications". Drug Target Insights, v. 2, n.2, pp. 147-157, Jul. 2007.

[14] BANIK, B. L., FATTAHI, P., BROWN, J. L.,"Polymeric nanoparticles: the future of nanomedicine". Nanomedicine and Nanobiotechnology, v. 8, n. 2, pp. 271-299, Aug. 2015.

[15] PASCOLI, M., et al. "State of the art of polymeric nanoparticles as carrier systems with agricultural applications: a minireview". Energy, Ecology and Environment, v. 3, n. 3, pp. 137-148, Apr. 2018.

[16] MAES, C., BOUQUILLON, S., FAUCONNIER, M.L.,"Encapsulation of Essential Oils for the Development of Biosourced Pesticides with Controlled Release: A Review". Molecules, v. 24, n. 14, pp. 1-17, Jul. 2019.

[17] FRANCISCO, E.V., GARCÍA-ESTEPA, R.M.,"Nanotechnology in the agrofood industry". Journal of Food Engineering, v. 238, n. 1, pp. 1-11, Dec. 2018.

[18] NASROLLAHZADEH, M., et al. "An Introduction to Nanotechnology". Interface Science and Technology, v. 28, n. 1, pp. 1-27, Jan. 2019.

[19] ESFANJANI, A.F., JAFARI, S.M.,"Nanoencapsulation of Phenolic Compounds and Antioxidants". Nanoencapsulation of Food Bioactive Ingredients, v. 1, n. 2, pp. 63-101, Jan. 2017.

[20] GHASEMISHAHRESTANI, Z., et al. "Tunable Synthesis of Gelatin Nanopartcles Employing Sophorolipid and Plant Extract, a Promising Drug Carrier". World Journal of Pharmacy and Pharmaceutical Sciences, v. 4, n. 7, pp. 1365-1381, May. 2015.

[21] SILVA, L.S., et al., "Encapsulation of Piper aduncum and Piper hispidinervum Essential Oils in Gelatin Nanoparticles- A Possible Sustainable Control Tool of A. aegypti, T. urticae and C. lataniae”. J Sci Food Agric., v. 99, n. 2, pp. 685-695, Jul. 2018.

[22] XIAO, Z., XU, Z., ZHU, G.,"Production and characterization of nanocapsules encapsulated linalool by ionic gelation method using chitosan as wall material". Food Science and Technology, v. 37, n. 3, pp. 613619, Oct. 2017.

[23] PAREDES, A.J., et al., "Nanoencapsulation in the food industry: manufacture, applications and characterization". Journal of Food Bioengineering and Nanoprocessing, v. 1, n. 1, p. 56-79, Mar. 2016.

[24] BASTO, S., et al. "Emulsão e microemulsão: novos sistemas de liberação controlada de fármacos no tratamento veterinário: Revisão". Medicina Veterinária (UFRPE), v. 10, n. 1-4, pp. 25-33, Dec. 2016.

[25] FRANZOL, A., REZENDE, M.C.,"Estabilidade de emulsões: um estudo de caso envolvendo emulsionantes aniônico, catiônico e não-iônico". Polímeros, v. 25, n. 1, pp. 1-9, Jan. 2015.

[26] DELGADO, J.M.F., "Preparação e Caracterização de Nanotransportadores (Nanocápsulas, Nanoesferas, Lipossomas e Transportadores Lipídicos Nanoestruturados) sem substância ativa", Dissertação de M.Sc., Instituto Politécnico de Bragança/Escola Superior de Tecnologia e Gestão, Goiânia, GO, Brasil, 2013.

[27] ANANDHARAMAKRISHNAN, C., "Characterization of Nanoparticles", In: Richard W. Hartel, Techniques for Nanoencapsulation of Food Ingredients, v. 8, Springer, pp. 65-67, 2014.

[28] FROIIO, F., et al., "Essential Oils-Loaded Polymer Particles: Preparation, Characterization and Antimicrobial Property". Polymers, v. 11, n. 6, pp. 1-15, Jun. 2019.

[29] OLIVEIRA, L.M., et al., "Alternative Biodefensive based on the Essential Oil fromAllium sativumEncapsulated in PCL/Gelatin Nanoparticles". Journal of Food Engineering and Technology, v. 8, n. 2, pp. 6574, Aug. 2019.

[30] NECAS, D., KLAPETEK, P., "Gwyddion: an open-source software for SPM data Analysis". Central European Journal of Physics, v. 10, n. 1, pp. 181-188, Feb. 2011.

[31] SCHNEIDER, C.A., RASBAND, W.S., ELICEIRI, K.W., "NIH Image to ImageJ: 25 years of image analysis". Nature Methods, v. 9, n. 7, pp. 671-675, Jun. 2012. 
[32] YANG, F.L. et al., "Structural Characterization of Nanoparticles Loaded with Garlic Essential Oil and Their Insecticidal Activity against Triboliumcastaneum (Herbst) (Coleoptera: Tenebrionidae)". Journal of Agricultural and Food Chemistry, v. 57, n. 21, pp. 10156 - 10162, Oct. 2009.

[33] BADRI, W., et al., "Poly ( $\varepsilon$-caprolactone) nanoparticles loaded with indomethacin and Nigella Sativa L. es-sential oil for the topical treatment of inflammation”. Journal of Drug Delivery Science and Technology, v. 46, n. 17, pp. 234-243, May. 2018.

[34] ETHIRAJAN, A., et al., "Synthesis and Optimization of Gelatin Nanoparticles Using the Miniemulsion Process". Biomacromolecules, v. 9, n. 9, pp. 2383-2389, Oct. 2008

[35] NAIDU, B.V.K., PAULSON, A.T., "A New Method for the Preparation of Gelatin Nanoparticles: Encapsulation and Drug Release Characteristics”. Journal of Applied Polymer Science, v. 121, n. 6, pp. 3495 3500, Sep. 2011.

[36] GADELMAWLA, E.S., KOURA, M.M., MAKSOUD, T.M.A., et al., "Roughness parameters", Journal of Materials Processing Technology, v.123, pp. 133-145. Apr. 2002.

\section{ORCID}

Ítalo Carvalho Costa

https://orcid.org/0000-0003-1858-8183

Sidney Gomes. Azevedo

https://orcid.org/0000-0001-8742-4399

Edgar Aparecido Sanches

https://orcid.org/0000-0002-1446-723X

Henrique Duarte da Fonseca Filho https://orcid.org/0000-0001-9811-6391 